\title{
Identification of immunological, inflammatory, hematological, and coagulation abnormalities associated with severity and mortality of COVID-19: a meta-analysis of 64 observational studies
}

\author{
Rundong Qin ${ }^{1}$, Li He ${ }^{1}$, Zhaowei Yang ${ }^{1}$, Nan Jia ${ }^{1}$, Ru-chong Chen ${ }^{1}$, Jiaxing Xie ${ }^{2}$, Wanyi \\ $\mathrm{Fu}^{1}$, Hao $\mathrm{Chen}^{1}$, Xinliu Lin ${ }^{1}$, Renbin Huang ${ }^{1}$, Tian Luo ${ }^{1}$, Yukai Liu ${ }^{1}$, Siyang Yao ${ }^{1}$, Mei \\ Jiang ${ }^{1}$, and Jing $\mathrm{Li}^{1}$ \\ ${ }^{1}$ Guangzhou Medical College First Affiliated Hospital \\ ${ }^{2}$ Guangzhou Institute of Respiratory Disease
}

September 11, 2020

\begin{abstract}
Background: Laboratory abnormalities associated with disease severity and mortality in patients with coronavirus disease 2019 (COVID-19) have been reported in many observational studies. However, there are significant heterogeneities in patient characteristics and research methodologies in these studies. Objectives: We aimed to provide an updated synthesis of the association between laboratory abnormalities and COVID-19 prognosis. Methods: We conducted an electronic search of PubMed, Scopus, Ovid, Willey, Web of Science, and the China National Knowledge Infrastructure (CNKI) for studies reporting hematological, coagulation, inflammatory, and immunological results during hospital admission of COVID-19 patients with different severities and outcomes. Results: A total of 64 studies were included in the current meta-analysis, with 8 hematological, 3 coagulation, 5 inflammatory, and 23 immunological variables reported. Of them, white blood cell (WBC) and neutrophil counts, D-dimer level, procalcitonin (PCT), erythrocyte sedimentation rate (ESR), C-reactive protein (CRP), ferretin, serum amyloid A (SAA), interleukins (ILs)-2R, IL-6, and IL-10 were significantly increased in severely ill patients and non-survivors. Meanwhile, nonseverely ill patients and survivors presented significantly higher counts of eosinophils, lymphocytes, and CD4+ and CD $8+\mathrm{T}$ cells. Conclusions: The current meta-analysis provides a comprehensive and updated synthesis of the association between admission laboratory abnormalities with severity and mortality of COVID-19. Our results highlight that increases in the levels of PCT, ESR, CRP, ferretin, SAA, IL-2R, IL-6, and IL-10 were associated with disease deterioration, whereas elevated eosinophils, lymphocytes, and T-cell subsets might serve as indicators of favorable outcomes.
\end{abstract}

\section{Hosted file}

Manuscript.docx available at https://authorea.com/users/355985/articles/480576identification-of-immunological-inflammatory-hematological-and-coagulationabnormalities-associated-with-severity-and-mortality-of-covid-19-a-meta-analysis-of64-observational-studies 